\title{
Compromiso Organizacional del Funcionario Municipal Rural de la Provincia de Nuble, Chile
}

\section{ORGANIZATION COMMITMENT OF RURAL MUNICIPAL WORKERS}

Dra. María M. Chiang Vega ${ }^{1}$, Nelly M. Gómez Fuentealba ${ }^{2}$ Lissette M. Wackerling Patiño ${ }^{3}$

1. Universidad del Bio-Bío. Concepción, Chile. e-mail: mchiang@ubiobio.cl

2. Universidad del Bío-Bío. Concepción, Chile. e-mail: ngomez@ubiobio.cl

3. Facultad de Ciencias Empresariales, Universidad del Bío-Bío, Concepción. Chile.

\begin{abstract}
RESUMEN
Introducción: La importancia del compromiso organizacional en el área pública es relevante al momento de analizar la disposición y el comportamiento hacia la tarea para el logro de los fines institucionales. Objetivo: Analizar los tipos de compromiso organizacional (afectivo, normativo y de continuación) basados en la teoría de Meyer y Allen (1991) presente en funcionarios de dos municipalidades chilenas comparando los tipos de compromiso entre éstas, según género y tipo de contrato. Materiales y método: La muestra es de 82 trabajadores, se realiza un estudio descriptivo no experimental de carácter transversal aplicando cuestionario para compromiso del trabajador de Meyer y Allen (1991). Resultados: Se encuentra una diferencia en los compromisos afectivo y normativo entre ambas instituciones y símil con respecto al compromiso de continuación. Con respecto al compromiso versus género, no existen diferencias estadísticamente significativas. En el análisis de relación entre compromiso versus tipo de contrato, el nivel de compromiso no varía según el tipo de contrato que tienen los funcionarios municipales.
\end{abstract}

(Chiang M, Gómez N, Wackerling L, 2016. Compromiso Organizacional del Funcionario Municipal Rural de la Provincia de Ñuble, Chile. Cienc Trab. May-Ago; 18 [56]: 134-138).

Palabras claves: COMPROMISO ORGANIZACIONAL, MUNICIPALIDADES, FUNCIONARIOS MUNICIPALES.

\section{ABSTRACT}

Introduction: The importance of organizational commitment in the public area is relevant when the layout and behaviour are analysed toward the corporate goals achievements. Objective: To Analyse the types of organizational commitment (affective, normative and continuance) based on the theory of Meyer and Allen (1991) present in two Chilean officials municipalities comparing the types of compromise between them, by gender and type of contract. Materials and Methods: The sample is comprised by 82 workers, a non-experimental descriptive cross-sectional study was made by using questionnaire commitment worker of Meyer and Allen (1991). Results: We found a difference in the affective and normative commitments between both institutions and a similar regarding the continuance commitment. Concerning commitment versus gender, no statistically significant differences were found. The analysis of relationship between commitment versus contract type, the commitment level does not vary depending on the type of contract that municipal workers have.

Keywords: ORGANIZATIONAL COMMITMENT, MUNICIPALITIES, MUNICIPAL WORKERS.

\section{INTRODUCCION}

En la actualidad el compromiso de las personas con la organización constituye un activo importante, puede ser uno de los mecanismos que tiene la dirección de recursos humanos para analizar la lealtad y vinculación de los empleados con su organización. Es, por tanto, importante para las organizaciones conocer no sólo el tipo sino también el grado de compromiso de sus miembros. ${ }^{1}$ Según Robbins ${ }^{2}$ el compromiso organizacional es la identificación del individuo con la organización que lo emplea; Harter, Schmidt

Correspondencia / Correspondence:

Lissette M. Wackerling

Tel.: +56 985276450

e-mail: wackerling@gmail.com

Recibido: 04 de Junio de 2016 / Aceptado: 24 de Junio de 2016 y Hayes ${ }^{3}$ consideran que el compromiso laboral incluye al denominado involucramiento de los individuos y la satisfacción y entusiasmo por el trabajo. El concepto de "compromiso laboral" se identifica en las empresas como el vínculo de lealtad por el cual el trabajador desea permanecer en la organización, debido a su motivación implícita. Compromiso como proceso de identificación y de creencia acerca de la importancia de su labor y la necesidad y utilidad de las funciones que realiza en el trabajo. ${ }^{4}$

En el concepto de compromiso laboral se incluyen la aceptación de las metas de la organización, la disposición a realizar esfuerzos en pro de ésta y la expresión del deseo de mantenerse como miembro activo de la misma, dentro de una práctica constante. Para Álvarez, Zancudo y Rivas ${ }^{5}$ el compromiso es un concepto que dice relación a la buena disposición del empleado o trabajador hacia el trabajo, a la buena voluntad, al sentido de lealtad con la empresa. Meyer \& Allen ${ }^{6}$ definieron el compromiso como un estado psicológico que caracteriza la relación entre una persona y una organización, la cual presenta consecuencias respecto a la decisión para continuar en la organización o dejarla. 
Este estudio se sostiene en la teoría de Meyer y Allen, la que se concentra en la división del compromiso en tres componentes: afectivo, de continuación y normativo; así, la naturaleza del compromiso es, respectivamente, el deseo, la necesidad o el deber permanecer en la organización; el compromiso afectivo (deseo) se refiere a los lazos emocionales que las personas forjan con la organización al percibir la satisfacción de sus necesidades (especialmente las psicológicas) y expectativas; por ende, disfrutan de su permanencia en la organización, el compromiso de continuación (necesidad): es muy posible que la persona evalúe las inversiones en tiempo y esfuerzo que perdería en caso de dejar la organización, o sea, los costos (financieros, físicos, psicológicos) en los cuales se incurriría al retirarse, o de las pocas posibilidades para encontrar otro empleo. Se refleja aquí una faceta calculadora, pues se refiere a la prosecución de inversiones (planes de pensiones, prima de antigüedad, aprendizaje, etc.) acumuladas y resultantes de la pertenencia a la organización y que dejarla resulta cada vez más costoso; el compromiso normativo (deber): en él se encuentra la creencia en la lealtad a la organización (sentido moral), quizá por recibir ciertas prestaciones (por ejemplo, capacitación o pagos de colegiaturas) conducente a un sentido del deber proporcionar una correspondencia. Este aspecto va en concordancia con la teoría de la reciprocidad: quien recibe algún beneficio adquiere el precepto moral interno de retribuir al donante. ${ }^{7}$ Estas modalidades de compromiso determinan una disposición diferente hacia la organización; las personas con alto compromiso afectivo se muestran deseosas de hacer esfuerzos en su desempeño, las personas con un compromiso de continuidad están necesitadas de permanecer en la empresa y las personas con compromiso normativo se sienten obligadas a permanecer.

Así entendido, el concepto de compromiso laboral ha cobrado importancia en las organizaciones, entre otras razones, debido al interés por entender qué retiene a los trabajadores en la organización. Entonces, se puede considerar el compromiso organizacional como la fuerza con la que un individuo se siente vinculado a una organización y que implica el seguimiento de un curso de acción relevante para ésta.

\section{MATERIALES Y MÉTODO}

\section{Objetivo}

Analizar los tipos de compromiso organizacional presentes en dos municipalidades rurales pertenecientes al sistema público chileno, comparando los tipos de compromiso entre municipalidades, según género y tipo de contrato. Para estos se identificarán las características relacionadas de cada funcionario y se determinará el tipo de compromiso organizacional con el que cada trabajador se identifica.

\section{Metodología}

Para el logro del objetivo se desarrolla un estudio descriptivo no experimental de carácter transversal.

Se plantean las siguientes hipótesis:

H1:Existen diferencias significativas en el grado de compromiso dependiendo de la municipalidad a la que pertenece el encuestado.

H2: Existen diferencias significativas en el grado de compromiso dependiendo el género del encuestado.

H3:Existen diferencias significativas en el grado de compromiso dependiendo del tipo de contrato del encuestado.

\section{Instrumento}

El instrumento de medición utilizado en este estudio corresponde al "Cuestionario del compromiso del trabajador con su organización" creado por Meyer y Allen ${ }^{6}$, compuesto por 21 preguntas cerradas en un formato de respuesta de Likert de cinco puntos (puntuación del 1 al 5). Este instrumento está orientado a medir los tres componentes de su teoria: afectivo, normativo y de continuación (costo/alternativa), y validado para muestras de trabajadores por Chiang, Salazar y Núñez. ${ }^{8}$ El cuestionario fue entregado a los funcionarios, la colaboración de los trabajadores fue voluntaria y anónima, la aplicación del cuestionario fue personal, autoaplicado y sin control de tiempo. Todos los sujetos recibieron la encuesta con una carta adjuntada donde se les explicaba los objetivos de la investigación y la confidencialidad de los datos entregados.

\section{Universo y muestra}

El universo de estudio lo constituyen los trabajadores de dos municipalidades rurales de la región del Bío-Bío, con un total de 88 funcionarios, se excluyeron 6 por lo que el análisis se realizó con 82 individuos con una tasa de respuesta del 93,1\%.

A este universo corresponden 34 mujeres y 48 hombres con una edad media de 34 años para funcionarias y 42 años para funcionarios y una antigüedad en su lugar de trabajo de 7,3 y 9 años respectivamente.

\section{RESULTADOS}

\section{a) Análisis de Fiabilidad}

Como método de fiabilidad se utilizará el alfa de Cronbach; este coeficiente expresa cuánto hay de relación en las respuestas, es decir, es un indicador global entre las respuestas.

Tabla 1.

Fiabilidad del compromiso organizacional.

$\begin{array}{lcccc}\text { No Variables } & \begin{array}{c}\text { N } \\ \text { de Items }\end{array} & \begin{array}{c}\text { Alfa de } \\ \text { Cronbach } \\ (2007)\end{array} & \begin{array}{c}\text { Alfa de } \\ \text { Cronbach } \\ \text { Chiang y otros } \\ (2010)\end{array} & \begin{array}{c}\text { Alfa de } \\ \text { Cronbach } \\ \text { Investigación } \\ \text { actual }\end{array} \\ \text { 1. Compromiso Afectivo } & 7 & 0,863 & 0,715 & 0,779 \\ \text { 2. Compromiso Normativo } & 6 & 0,785 & 0,797 & 0,702 \\ \text { 3. Compromiso de Continuidad } & 8 & 0,7 & 0,854 & 0,789\end{array}$

Fuente: Elaboración propia.

La Tabla № 1 muestra que la puntuación Alfa para las escalas correspondientes a Compromiso organizacional en una muestra con estas características, se encuentran entre los valores 0,702 y 0,789. Esto implica que para todas las escalas, el coeficiente de fiabilidad que representan es aceptable. Es decir, el instrumento posee todas las cualidades para ser aplicado y posteriormente analizado en esta muestra.

Tabla 2.

Promedios compromiso organizacional.

\begin{tabular}{|c|c|c|c|c|c|}
\hline $\mathrm{N}^{\circ}$ Factores & $\begin{array}{r}\text { Municip } \\
\text { Femenino }\end{array}$ & $\begin{array}{l}\text { idad } 1 \\
\text { Masculino }\end{array}$ & $\begin{array}{r}\text { Munic } \\
\text { Femenin }\end{array}$ & $\begin{array}{l}\text { alidad } 2 \\
\text { Masculino }\end{array}$ & $\begin{array}{l}\text { Media } \\
\text { Total }\end{array}$ \\
\hline 1. Compromiso Afectivo & 3,95 & 3,75 & 3,51 & 3,69 & 3,73 \\
\hline 2. Compromiso Normativo & 3,67 & 3,71 & 3,34 & 3,51 & 3,56 \\
\hline 3. Compromiso de Continuidad & 3,50 & 3,58 & 3,39 & 3,74 & 3,55 \\
\hline
\end{tabular}

Fuente: Elaboración propia.

\section{b) Análisis de promedios de Compromiso Organizacional}

En la Tabla $\mathrm{N}^{\circ} 2$, de promedios del compromiso organizacional (con un rango entre 1 y 5) se observa que:

1. Compromiso afectivo promedia un valor de 3,73, el valor más 
bajo se encuentra en las mujeres de la municipalidad 2 con un 3,51 y el valor más alto se encuentra en las mujeres de la municipalidad 1 con un 3,95.

2. Compromiso normativo promedia un valor de 3,56 , el valor más bajo se encuentra en mujeres de la municipalidad 2 con un 3,34 y el valor más alto se encuentra en hombres de la municipalidad 1 con un 3,71.

3. Compromiso de continuidad promedia un valor de 3,55 , el valor más bajo pertenece a mujeres de la municipalidad 2 con un 3,39 y el valor más alto a hombres de la municipalidad 2 con un 3,74 .

\section{c) Análisis comparativo}

En la siguiente sección se presentan las pruebas de hipótesis realizadas en la muestra y los correspondientes resultados para comparar los grados medios o medianos de compromiso organizacional por municipalidad. La comparación compromiso versus género y compromiso versus tipo de contrato se realiza para la muestra completa.

Para los correspondientes análisis, se aplica el Test de Shapiro Wilk, que es una prueba de hipótesis para verificar la normalidad de los datos en muestras pequeñas de entre 3 y 50 datos u observaciones. ${ }^{9}$ Para comprobar el cumplimiento de homogeneidad de varianzas, se utiliza el test de Bartlett que contrasta la hipótesis nula de que las $\mathrm{k}$ muestras provienen de la misma población o de poblaciones idénticas. ${ }^{10}$ Para la comparación de medias se aplica el test -T cuando se cumplen los supuestos de normalidad y homogeneidad de varianzas. ${ }^{11}$ En caso contrario, se utiliza el test no paramétrico de WilcoxonMann-Whitney, esta prueba es útil para evaluar si dos grupos independientes fueron extraídos de la misma población, constituye una opción a la prueba paramétrica t cuando no se verifican los supuestos o cuando las mediciones de la investigación se encuentran en una escala inferior a la de intervalo. ${ }^{12}$

Para la comparación entre los tres tipos de contratos, se utiliza análisis de varianza y el procedimiento de Tukey para las comparaciones pareadas cuando se rechaza la hipótesis nula de que las tres medias son iguales. ${ }^{13} 0$ la prueba de Kruskal-Wallis cuando no se cumple la normalidad de las poblaciones. ${ }^{12}$

\section{i) Compromiso versus municipalidad}

La normalidad de los datos y homogeneidad de varianzas, se estudiaron mediante el test de Shapiro-Wilks (SW) y el test de Bartlett respectivamente; la Tabla No 3 muestra los resultados que permiten concluir normalidad en las tres variables de compromiso (valor $-\mathrm{p}>0,1)$ y homogeneidad de varianzas (valor $-\mathrm{p}>0,05$ ). Los correspondientes resultados del test $-\mathrm{T}$ de comparación de medias arrojan diferencias estadísticamente significativas en el compromiso afectivo (Test $-\mathrm{t}=3,21$, valor $-\mathrm{p}<0,01$ ) y compromiso normativo (Test $-\mathrm{T}=2,03$, valor $-\mathrm{p}<0,05$ ). No se encon-

Tabla 3.

Test de normalidad y homogeneidad de varianzas.

\begin{tabular}{|c|c|c|c|c|c|}
\hline Compromiso & Municipalidad & $\begin{array}{l}\text { Norma } \\
\text { Test Shapiro } \\
\text { Wilks }\end{array}$ & $\begin{array}{l}\text { alidad } \\
\text { Valor -p }\end{array}$ & $\begin{array}{c}\text { lgualdad d } \\
\text { Test de } \\
\text { Bartlett }\end{array}$ & $\begin{array}{l}\text { varianzas } \\
\text { Valor }-p\end{array}$ \\
\hline Afectivo & $\begin{array}{l}1 \\
2\end{array}$ & $\begin{array}{l}0,97 \\
0,97\end{array}$ & $\begin{array}{l}0,29 \\
0,29\end{array}$ & 3,17 & 0,07 \\
\hline Normativo & $\begin{array}{l}1 \\
2\end{array}$ & $\begin{array}{l}0,97 \\
0,98\end{array}$ & $\begin{array}{l}0,24 \\
0,72\end{array}$ & 1,05 & 0,31 \\
\hline Continuación & $\begin{array}{l}1 \\
2\end{array}$ & $\begin{array}{l}0,97 \\
0,98\end{array}$ & $\begin{array}{l}0,31 \\
0,34\end{array}$ & 0,06 & 0,81 \\
\hline
\end{tabular}

Fuente: Elaboración propia.
Tabla 4.

Test de comparación de medias.

\begin{tabular}{lc|cc} 
& & \multicolumn{2}{|c}{ Comparación de Medias } \\
Compromiso & Municipalidad & Test $-\mathrm{t}$ & Valor $-\mathrm{p}$ \\
\hline Afectivo & 1 & 3,21 & 0,001 \\
Normativo & 2 & & \\
Continuación & 1 & 2,03 & 0,045 \\
& 2 & & \\
& 1 & $-0,34$ & 0,73
\end{tabular}

Fuente: Elaboración propia.

Gráfico 1.

Comparación del Compromiso vs Municipalidad.

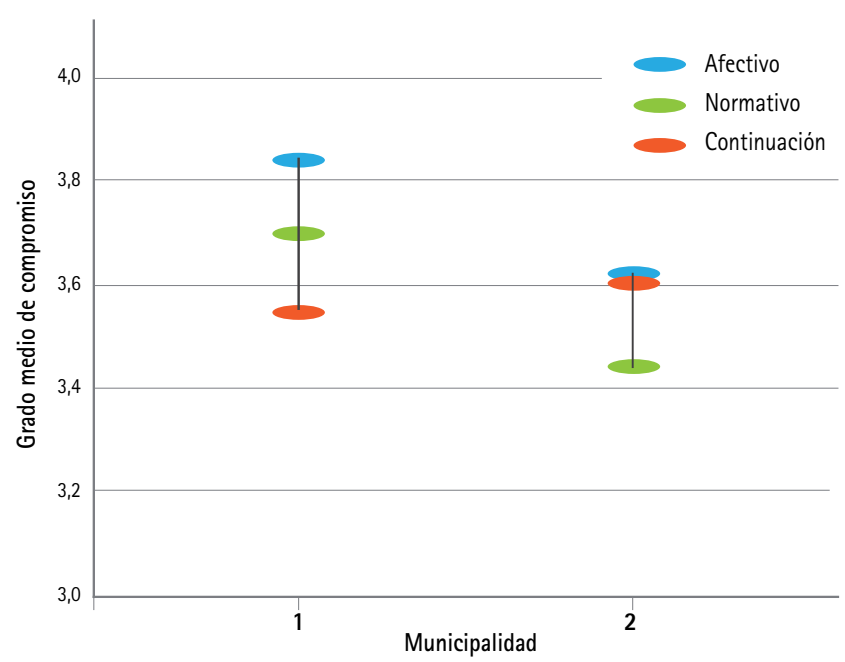

traron diferencias significativas entre municipalidades en el compromiso de continuación (Test $-\mathrm{T}=-0,34$, valor $-\mathrm{p}>0,05$ ) como se observa en la Tabla $\mathrm{N}^{\circ} 4$.

El Gráfico $\mathrm{N}^{\circ} 1$ muestra el grado medio de cada tipo de compromiso por municipalidad, donde se observan marcadas diferencias para el compromiso afectivo y normativo entre las instituciones.

En el caso del compromiso afectivo se observa que los funcionarios poseen un mayor apego emotivo hacia su institución de trabajo.

Respecto al compromiso normativo también existe un grado mayor de compromiso en la municipalidad 1, probablemente esta institución posee mejor organización y medidas más claras que permiten desempeñarse mejor a los trabajadores.

Por último, en el compromiso de continuación existe una diferencia mínima favorable a la municipalidad 2.

\section{ii) Compromiso versus género}

Las variables de compromiso afectivo y de continuación cumplen con los requisitos de normalidad y homogeneidad de varianzas tanto para la muestra masculina como femenina. Como se desprende de la Tabla № 5, no se encuentra evidencia suficiente para rechazar las correspondientes hipótesis (valor $-\mathrm{p}>0,05$ ). En relación al compromiso normativo, no es claro el cumplimiento del supuesto de homogeneidad de varianzas (Test de Bartlett $=3,83$, valor $-p=0,048$ ), por lo que se aplica el test - T de varianzas distintas al momento de comparar los niveles medios de compromisos entre hombres y mujeres.

Como se muestra en la Tabla No 5 , el test - T de comparación de medias, no arroja diferencias estadísticamente significativas entre hombres y mujeres respecto de los tres tipos de compromiso, en todos los casos el valor - p es superior al 10\%. 
Tabla 5 .

Test de normalidad y homogeneidad de varianzas.

\begin{tabular}{|c|c|c|c|c|c|}
\hline Compromiso & Género & $\begin{array}{l}\text { Normal } \\
\text { Test Shapiro } \\
\text { Wilks }\end{array}$ & $\begin{array}{l}\text { alidad } \\
\text { Valor -p }\end{array}$ & $\begin{array}{l}\text { Igualdad d } \\
\text { Test de } \\
\text { Bartlett }\end{array}$ & varianzas \\
\hline Afectivo & $\begin{array}{l}\text { Femenino } \\
\text { Masculino }\end{array}$ & $\begin{array}{l}0,94 \\
0,96\end{array}$ & $\begin{array}{l}0,06 \\
0,10\end{array}$ & 0,06 & 0,80 \\
\hline Normativo & $\begin{array}{l}\text { Femenino } \\
\text { Masculino }\end{array}$ & $\begin{array}{l}0,96 \\
0,97\end{array}$ & $\begin{array}{l}0,32 \\
0,21\end{array}$ & 3,83 & $0,048^{*}$ \\
\hline Continuación & $\begin{array}{l}\text { Femenino } \\
\text { Masculino }\end{array}$ & $\begin{array}{l}0,97 \\
0,97\end{array}$ & $\begin{array}{l}0,34 \\
0,20\end{array}$ & 1,49 & 0,22 \\
\hline
\end{tabular}

Fuente: Elaboración propia.

Tabla 6.

Test - T de comparación de medias.

\begin{tabular}{ll|cc} 
Compromiso & Género & \multicolumn{2}{|c}{ Comparación de Medias } \\
Afectivo & $\begin{array}{l}\text { Femenino }-\mathrm{t} \\
\text { Masculino }\end{array}$ & 0,17 & Valor $-\mathrm{p}$ \\
Normativo & $\begin{array}{l}\text { Femenino } \\
\text { Masculino }\end{array}$ & $-0,666^{*}$ & 0,51 \\
Continuación & $\begin{array}{l}\text { Femenino } \\
\text { Masculino }\end{array}$ & $-1,30$ & 0,20 \\
*se aplicó test -T de varianzas distintas. & Fuente: Elaboración propia.
\end{tabular}

iii) Compromiso versus tipo de contrato

Tanto el compromiso afectivo como el normativo cumplen con la normalidad y homogeneidad de varianzas en relación a los tipos de contrato (valor $-\mathrm{p}>0,05$ ). El compromiso de continuación cumple con la normalidad, pero la variabilidad entre los tipos de contrato es diferente (valor $-\mathrm{p}<0,05$ ), por este motivo se aplica el análisis de varianza en los dos primeros tipos de compromiso y test no paramétrico de Kruskal-Wallis para el compromiso de continuación. Los resultados correspondientes se presentan en las tablas № 7 y 8.

Tabla 7.

Test de normalidad y homogeneidad de varianzas.

\begin{tabular}{|c|c|c|c|c|c|}
\hline \multirow[b]{2}{*}{ Compromiso } & \multirow[b]{2}{*}{ Tipo de contrato } & \multicolumn{2}{|c|}{ Normalidad } & \multicolumn{2}{|c|}{ Igualdad de varianzas } \\
\hline & & $\begin{array}{l}\text { Test Shapiro } \\
\text { Wilks }\end{array}$ & Valor -p & $\begin{array}{l}\text { Test de } \\
\text { Bartlett }\end{array}$ & Valor $-p$ \\
\hline \multirow{3}{*}{ Afectivo } & Planta & 0,96 & 0,54 & \multirow{3}{*}{1,77} & \multirow{3}{*}{0,41} \\
\hline & Contrata & 0,93 & 0,28 & & \\
\hline & Honorario & 0,99 & 0,89 & & \\
\hline \multirow{3}{*}{ Normativo } & Planta & 0,96 & 0,38 & \multirow{3}{*}{2,07} & \multirow{3}{*}{0,35} \\
\hline & Contrata & 0,94 & 0,39 & & \\
\hline & Honorario & 0,98 & 0,80 & & \\
\hline \multirow{3}{*}{ Continuación } & Planta & 0,94 & 0,19 & \multirow{3}{*}{7,93} & \multirow{3}{*}{0,02} \\
\hline & Contrata & 0,96 & 0,74 & & \\
\hline & Honorario & 0,98 & 0,80 & & \\
\hline
\end{tabular}

Fuente: Elaboración propia.

Tabla 8.

Análisis de varianza.

\begin{tabular}{|c|c|c|c|c|c|}
\hline \multirow{2}{*}{$\begin{array}{l}\text { Compromiso } \\
\text { Afectivo }\end{array}$} & \multicolumn{5}{|c|}{ Prueba ANOVA } \\
\hline & GI & $\begin{array}{l}\text { Suma de } \\
\text { cuadrados }\end{array}$ & $\begin{array}{l}\text { Cuadrados } \\
\text { medios }\end{array}$ & $\mathrm{F}$ & sig. \\
\hline Tipo de contrato & 2 & 2,32 & 1,16 & 3,47 & 0,04 \\
\hline Residuos & 79 & 26,42 & 0,33 & & \\
\hline Normativo & $\mathrm{Gl}$ & $\begin{array}{l}\text { Suma de } \\
\text { cuadrados }\end{array}$ & $\begin{array}{l}\text { Cuadrados } \\
\text { medios }\end{array}$ & $\mathrm{F}$ & sig. \\
\hline Tipo de contrato & 2 & 0,19 & 0,10 & 0,28 & 0,75 \\
\hline \multirow[t]{2}{*}{ Residuos } & 79 & 26,90 & 0,34 & & \\
\hline & \multicolumn{5}{|c|}{ Test de Kruskal-Wallis } \\
\hline \multirow[t]{2}{*}{ Continuación } & $\mathrm{GI}$ & $\mathrm{K}-\mathrm{W}$ & sig. & & \\
\hline & 2 & 2,31 & 0,31 & & \\
\hline
\end{tabular}

Fuente: Elaboración propia.
Tabla 9.

Prueba de tukey para comparaciones pareadas.

$\begin{array}{lcccc}\text { Tipo de contrato } & \text { Diferencia } & \text { IC inf } & \text { IC sup } & \text { Sig } \\ \text { Contrata - Planta } & -0,1 & -0,57 & 0,37 & 0,87 \\ \text { Honorario - Planta } & -0,37 & -0,73 & -0,01 & 0,04 \\ \text { Honorario - Contrata } & -0,27 & -0,69 & 0,15 & 0,27 \\ \text { Fuente: Elaboración propia. } & & & & \end{array}$

No se observan diferencias significativas en el grado de compromiso normativo en relación al tipo de contrato que posee el encuestado (valor $-p=0,75>0,05$ ), como tampoco en el compromiso de continuación $(\mathrm{K}-\mathrm{W}=2,31$, valor $-\mathrm{p}=0,31>$ $0,05)$. Mientras que para el compromiso afectivo se encuentran diferencias estadísticamente significativas entre tipo de contrato (valor $-\mathrm{p}=0,04<0,05$ ). Mediante la aplicación de la prueba de Tukey, se encuentra que el grado de compromiso afectivo difiere entre aquellos que tienen contrato a honorarios, y los que tienen contrato de planta. Como se observa en la Tabla $n^{\circ}$ 9, tanto el limite inferior como superior del intervalo de 95\% de confianza son negativos, lo que nos permite inferir que aquellos funcionarios que tienen contrato a honorarios declaran un grado de compromiso de continuación inferior en relación a los que tienen un contrato de planta, el valor - p de la prueba inferior a 0,05 nos lleva a la misma conclusión.

\section{DISCUSION}

\section{Conclusiones:}

Hipótesis $N^{\circ} 1$ : Respecto del tipo de compromiso organizacional presente en cada municipalidad, se observa que existe una diferencia con respecto al compromiso afectivo y normativo entre ambas instituciones y grado medio similar en ambos municipios con respecto al compromiso de continuidad. Esto puede significar en el caso del compromiso afectivo, a que éste responde directamente al sentimiento que provoca la institución en su persona y si se encuentra agradado de trabajar ahí. En esta investigación se obtiene un alto índice en ambas instituciones, por lo que los funcionarios públicos que trabajan en dicha entidad se sienten sinceramente comprometidos con su trabajo, logrando afecto con la institución.

En el compromiso normativo existe un sentimiento de reciprocidad a lo que la institución le entrega, similar en los dos organismos, por lo que los funcionarios se sienten con el deber de corresponder a lo que la institución les entrega (bonificaciones, capacitaciones, etc.); este compromiso obtiene igualmente un alto indice en ambas instituciones, por lo que esto se traduce a que las dos entidades poseen una número suficiente de beneficios dirigidos a sus empleados, lo que hace que éstos se sientan agradecidos con la institución por las gratificaciones que reciben al pertenecer a dicha entidad y esto se traduzca finalmente en el compromiso de ellos hacia su lugar de trabajo.

En definitiva, se presentan diferencias estadisticamente significativas entre las instituciones en el compromiso afectivo y normativo, es decir en dos de los tres tipos de compromiso, lo que hace a la variable municipalidad un factor preponderante en el grado de compromiso de los encuestados, con ciertas medidas, ambiente laboral, organización, etc. con certeza mejores en una que en la otra.

Respecto al compromiso de continuidad se entiende que existe una necesidad por parte del funcionario en seguir trabajando en 
su respectiva institución, ya que existen costos de dejarla y las pocas posibilidades reales de encontrar trabajo en otro lugar. En este caso los índices de compromiso de continuidad son altos, por lo que se desprende que existe un gran número de empleados que poseen una antigüedad laboral importante en ambas instituciones. Este tipo de compromiso es incentivado por la institución, ya que en muchas oportunidades el compromiso se logra sólo por el factor de años de trabajo e inversión obtenida por parte de los funcionarios. No se presentan diferencias significativas entre ambas municipalidades, por tanto, se entiende que este tipo de compromiso no varía según municipio. Se podría concluir que una mayoría de trabajadores con un alto índice de años de permanencia implicaría la existencia de un alto nivel de compromiso de continuidad en la entidad debido a este factor, que es transversal a la institución.

Hipótesis $N^{\circ} 2$ : Respecto al compromiso versus género, no existen diferencias estadísticamente significativas entre ambos géneros para esta investigación, por lo que se puede concluir que no existe diferencia en cuanto al nivel de compromiso entre funcionarias y funcionarios.

Hipótesis $N^{\circ}$ 3: Respecto a si existen diferencias significativas en relación entre compromiso versus tipo de contrato, se encuentra que el nivel de compromiso varía según el tipo de contrato, entendiendo que existen 3 tipos de contrato en el sistema público chileno; planta, contrata y honorarios, y que quienes tienen contrato tipo "planta" tienen un nivel muy alto de años trabajando en su puesto de trabajo; los contratos a "contrata", un nivel medio de tiempo trabajado en dicho puesto; y, los contratos a "honorarios", un índice bajo de años trabajando en su actual puesto laboral.
De este análisis se puede concluir que en el compromiso afectivo se muestra un nivel medio en aquellos funcionarios contratados bajo la modalidad planta, un índice alto para los contratados a contrata y un nivel bajo para quienes están contratados a honorarios. Los empleados con un mayor índice de compromiso afectivo son quienes están contratados a contrata y con un menor indice aquellos que están contratados a honorarios; esto puede deberse a que son estos funcionarios los que llevan menor tiempo trabajando, por lo que no logran aún establecer un vínculo con la institución que los haga sentirse comprometidos con ella.

En cuanto al compromiso normativo, se encuentra que los funcionarios contratados bajo la modalidad de planta y a contrata poseen un indice alto de este tipo de compromiso y aquellos que están contratados a honorarios un nivel bajo; esto puede ser debido a que los primeros llevan más años trabajando y, por ende, han apreciado en plenitud los beneficios que les entrega la institución a diferencia de aquellos que llevan menos tiempo trabajando en la entidad.

Respecto del compromiso de continuidad, los funcionarios contratados a planta son quienes tienen el mayor índice de este compromiso a diferencia de los otros dos tipos de contrato, los que tienen un indice bajo de este. Esto debido a que son los empleados que llevan un mayor número de años trabajando en el mismo lugar los que tienen mayor necesidad de seguir trabajando en su respectiva institución, ya que es muy poco rentable para ellos abandonarla, no en cambio para quienes llevan menos tiempo de trabajo en su puesto actual.

\section{Agradecimientos}

Los investigadores agradecen a los alcaldes y funcionarios municipales que hicieron posible este estudio. Proyecto de investigación 131018 3/R Universidad del Bío-Bío, Concepción, Chile.

\section{REFERENCIAS}

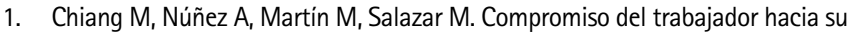
organización y la relación con el clima organizacional: un análisis de género y edad. Panorama socioeconómico. 2010;28(40):92-103.

2. Robbins $S$, Judge T. Comportamiento organizacional. $13^{\mathrm{a}}$ ed. México: Pearson; 2009.

3. Harter J, Schmidt F, Hayer T. Bussines unit level relationship between employee satisfaction, employee engagement and bussines outcomes a meta-analysis. APA PsycNet for institutions by the American Psicological association. J Appl Psycol. 2002;87(2): 268-279.

4. Álvarez de Mon S, Cardona P, Chinchilla M, Pérez J, Pin J, Poelmanss S, Rodríguez J, Torres M. Paradigmas del liderazgo. 2a ed. Madrid: McGraw-Hill; 2001.

5. Álvarez G, Zancudo, M T, Rivas A. El constructo clima organizacional: concepto, teorias, investigaciones y resultados relevantes. RIPO. 1992;11(2):25-50.

6. Meyer JP, Allen N. A three component conceptualization of organizational commitment. Hum Resour Manage R. 1991;1(1):61-89.
7. Gouldner AW. The norm of reciprocity: a preliminary statement. Am Sociol Rev. 1960;25(2):161-178.

8. Chiang M, Salazar M, Huerta P, Núñez A. Clima organizacional y satisfacción laboral en organizaciones del sector estatal. Desarrollo, adaptación y validación de instrumentos. Universum. 2008;2(23):66-85.

9. De la Garza J, Morales B, González B. Análisis estadistico Multivariante. Un enfoque teórico y práctico. México, DF: McGraw-Hill. 2012.

10. Montgomery D. Design and analysis of experiments. 3a ed. New York: Wiley; 1991.

11. Lapin L. Probability and Statistics for Modern Engineering. 2a ed. Boston: PWS-Kent; 1990.

12. Siegel $S$, Castellan J. Estadistica no paramétrica aplicada a las ciencias de la conducta. 4a ed. México: Trillas; 1995.

13. Walpole R, Myers R, Myers S. Probabilidad y Estadistica para Ingenieros. $6^{\mathrm{a}}$ ed. México: Prentice-Hall; 1999. 